\title{
CERTAIN REITERATION AND EQUIVALENCE RESULTS FOR THE COBOS-PEETRE POLYGON INTERPOLATION METHOD
}

\author{
STEFAN ERICSSON
}

\begin{abstract}
We prove some reiteration formulas for the Cobos-Peetre polygon method for $n+1$-tuples that consists of spaces $A_{i}$ where $A_{i}$ is of class $\theta_{i}$ with respect to a compatible pair $(X, Y)$. If $\theta_{i}$ is suitably chosen the $J$ - and $K$-method coincides and is equal to a space $(X, Y)_{\nu, q}$. For arbitrarily chosen $\theta_{i}$ the $J$ - and $K$-spaces will not, in general, coincide. In particular we show that interpolation of Lorentz spaces over the unit square yields that the $K$-space is the sum of two Lorentz spaces whereas the $J$-space is the intersection of the same two Lorentz spaces.
\end{abstract}

\section{Introduction}

In the theory of interpolation one usually interpolates between two Banach spaces. This area is well developed, see e.g. the bibliography by Maligranda [21], including approximately 2500 references. However, there are interpolation methods for families of Banach spaces, even infinite families, see e.g. the review and references given in [5].

This paper deals with the Cobos-Peetre polygon method. A method introduced by F. Cobos and J. Peetre, see [13], and further developed in [7], [12], [8], [16], [6], [11], [9] and [10]. There are other different real methods for interpolating between a finite collection of Banach spaces. We mention the $N$-space method of Sparr, see [22], the $2^{N}$-space method of Fernandez, see [15].

The main theorems are in Section 4. In that section we first consider $(n+1)$-tuples $\left(A_{0}, \ldots, A_{n}\right)$ on an arbitrary convex polygon, where $A_{i}$ is of class $\mathrm{C}\left(\theta_{i}, X, Y\right)$ for a compatible couple $(X, Y)$. If $\theta_{i}$ satisfies, for some $\delta \neq 0$ and $\rho \neq 0$,

$$
\theta_{i}=\delta x_{i}+\rho y_{i}, \text { for } i=0, \ldots, n,
$$

where $\left[\left(x_{0}, y_{0}\right)\left(x_{1}, y_{1}\right) \ldots\left(x_{n}, y_{n}\right)\right], y_{i} \geq 0$, is the underlying polygon, then it turns out that the $J$ - and $K$-spaces coincide and are equal to a real inter-

Received February 17, 1997. 
polation space $(X, Y)_{v, q}$. This will not, in general, be true if for some $i$ equation (1) does not hold. See Theorem 3. After that we consider more general choices of $\theta_{i}$. It turns out that the $K$-space will be a sum of two real interpolation spaces between $X$ and $Y$ whereas the corresponding $J$-space will be the intersection of the same two spaces, see Theorem 5 .

In particular these theorems show that there are, in most cases, no equivalence between the $K$ - and $J$-spaces. This is in wide contrast to the recent result of I.U. Asekritova and N.Ya. Krugljak, [1], that the $K$ - and $J$ spaces are equivalent for function lattices in the Sparr method case.

Section 5 deals with the Fernandez method for four spaces, i.e. when the polygon is the unit square. See Theorem 6. We give examples for Lorentz and weighted Lebesgue spaces.

One more reiteration result is presented in the last section. Generally, we there only get embeddings. But for special tuples we can reverse the embedding and thus obtain equivalence.

In order not to disturb our discussion later on and for the reader's convenience we have presented some basic facts concerning the classical real interpolation and the Cobos-Peetre polygon method in Section 2.

Notations: Two real-valued functions $f$ and $g$ are said to be equivalent, denoted by $f \approx g$, if there exists positive constants $C$ and $D$ such that

$$
C f(x) \leq g(x) \leq D f(x),
$$

for all $x$. By $f \lesssim g$ we mean that there exists a positive constant $C$ such that

$$
f(x) \leq C g(x),
$$

for all $x$. We also use the notation $A \cong B$ when $A$ and $B$ are equal as vector spaces and their norms are equivalent, i.e. they are identical as topological vector spaces.

\section{Preliminaries}

In this section we will briefly describe some well-known real interpolation methods between two and several spaces.

Let $\bar{A}:=\left(A_{0}, A_{1}\right)$ be a compatible Banach pair, i.e. two Banach spaces both linearly and continuously embedded in a Hausdorff topological vector space $A$.

The $K$ functional is defined for all $f \in A_{0}+A_{1}$ and $t>0$ by

$$
K(t, f, \bar{A}):=\inf \left\{\left\|f_{0}\right\|_{A_{0}}+t\left\|f_{1}\right\|_{A_{1}}: f_{0} \in A_{0}, f_{1} \in A_{1} \text { and } f=f_{0}+f_{1}\right\} .
$$

The $J$ functional is defined for all $u \in A_{0} \cap A_{1}$ and $t>0$ by 


$$
J(t, u, \bar{A}):=\max \left\{\|u\|_{A_{0}}, t\|u\|_{A_{1}}\right\} .
$$

One defines the $K$-interpolation space $\bar{A}_{\theta, q ; K}$, for $0<\theta<1$ and $1 \leq q \leq \infty$, as the set of $f \in A_{0}+A_{1}$ for which

$$
\|f\|_{\theta, q ; K}:=\left(\int_{0}^{\infty}\left(t^{-\theta} K(t, f, \bar{A})\right)^{q} \frac{d t}{t}\right)^{\frac{1}{q}}<\infty .
$$

The function $f \mapsto\|f\|_{\theta, q ; K}$ is used as a norm on this space. One also defines the $J$-interpolation space $\bar{A}_{\theta, q ; J}$, for $0<\theta<1$ and $1 \leq q \leq \infty$, as the set of $f \in A_{0}+A_{1}$ for which

$$
\|f\|_{\theta, q ; J}:=\inf \left(\int_{0}^{\infty}\left(t^{-\theta} J(t, u(t), \bar{A})\right)^{q} \frac{d t}{t}\right)^{\frac{1}{q}}<\infty .
$$

The infimum is taken over all representations of $f$ on the form

$$
f=\int_{0}^{\infty} u(t) \frac{d t}{t}
$$

In the formula (2) $u$ is strongly measurable with values in $A_{0} \cap A_{1}$ and the integral is understood as an intersection valued Bochner integral over $[a, b]$ $\subset(0, \infty)$ and convergence, $a \rightarrow 0$ and $b \rightarrow \infty$, in $A_{0}+A_{1}$ with norm $K(1, \cdot, \bar{A})$. The function $f \mapsto\|f\|_{\theta, q ; J}$ is used as a norm on this space.

One realizes that it is of great importance to determine the $K$ functional. It has almost become an art in itself to calculate it, see e.g. [2], [20].

The so called Equivalence Theorem states that $\bar{A}_{\theta, q ; K} \cong \bar{A}_{\theta, q ; J}$. This theorem is of fundamental importance. In view of this and since we only will deal with spaces up to equivalence we may, and will, omit the subscripts $K$ and $J$ and just writing $\bar{A}_{\theta, q}$.

An intermediate space, $X$, with respect to $\bar{A}$ is a Banach space for which $\Delta(\bar{A}) \hookrightarrow X \hookrightarrow \Sigma(\bar{A})$, here $\Delta(\bar{A}):=A_{0} \cap A_{1}$ with norm $J(1, \cdot, \bar{A})$ and $\Sigma(\bar{A}):=A_{0}+A_{1}$ with norm $K(1, \cdot, \bar{A})$. An intermediate space $X$ is said to be of class $\mathrm{C}_{K}(\theta, \bar{A})$ if

$$
K(t, f, \bar{A}) \lesssim t^{\theta}\|f\|_{X},
$$

for all $f \in X$ and of class $\mathrm{C}_{J}(\theta, \bar{A})$ if

$$
t^{\theta}\|u\|_{X} \lesssim J(t, u, \bar{A}),
$$

for all $u \in A_{0} \cap A_{1}$. Here $0 \leq \theta \leq 1$. If $X$ is both of classes $\mathrm{C}_{K}(\theta, \bar{A})$ and $\mathrm{C}_{J}(\theta, \bar{A})$ it is said to be of class $\mathrm{C}(\theta, \bar{A})$. For $0<\theta<1$ one can prove that an intermediate space $X$ is of class $\mathrm{C}(\theta, \bar{A})$ if and only if

$$
\bar{A}_{\theta, 1} \hookrightarrow X \hookrightarrow \bar{A}_{\theta, \infty}
$$


This means, in particular, that $\bar{A}_{\theta, q}$ is of class $\mathrm{C}(\theta, \bar{A})$.

The classical Reiteration Theorem states: If $\left(A_{0}, A_{1}\right)$ is a compatible pair of Banach spaces, $X_{i}$ of class $\mathrm{C}\left(\theta_{i}, \bar{A}\right)$ for $0 \leq \theta_{i} \leq 1, i=1,2$, and $\theta_{0} \neq \theta_{1}$ then

$$
\left(X_{0}, X_{1}\right)_{\eta, q} \cong\left(A_{0}, A_{1}\right)_{(1-\eta) \theta_{0}+\eta \theta_{1}, q},
$$

for $1 \leq q \leq \infty$ and $0<\eta<1$.

For an introduction to the theory of interpolation between two spaces see e.g. the books [2], [3], [4], [19].

We will now briefly discuss an extension of the real method described above to the case when interpolating between several Banach spaces. The method described below is the so called polygon method or Cobos-Peetre method which was introduced in [13].

If $A_{0}, \ldots, A_{n}$ are $n+1$ Banach spaces which are linearly and continuously embedded in a Hausdorff topological vector space $\mathrm{A}$, then $\left(A_{0}, \ldots, A_{n}\right)$ is said to be a compatible $(n+1)$-tuple, which we also denote by $\bar{A}$. Here and below $n \geq 2$.

Consider a convex polygon $\Pi:=\left[P_{0} \ldots P_{n}\right]$ in $R^{2}$, with vertices $P_{i}:=\left(x_{i}, y_{i}\right), i=0, \ldots, n$. Let $\bar{A}$ be a compatible $(n+1)$-tuple. Each space $A_{i}$ is thought of as sitting on the vertex $P_{i}$. We will now describe a way of creating interpolation spaces in each point in the interior of $\Pi$.

If $t>0, s>0$ and $f \in A_{0}+\ldots+A_{n}$, we define the $K$ functional by

$$
K(t, s, f, \bar{A}):=\inf \left\{\sum_{i=0}^{n} t^{x_{i}} s^{y_{i}}\left\|f_{i}\right\|_{A_{i}}: f=\sum_{i=0}^{n} f_{i}, f_{i} \in A_{i}\right\},
$$

and the $J$ functional, for $u \in A_{0} \cap \ldots \cap A_{n}$, by

$$
J(t, s, u, \bar{A}):=\max \left\{t^{x_{i}} s^{y_{i}}\|u\|_{A_{i}}: i=0, \ldots, n\right\} .
$$

Let $(\alpha, \beta)$ be a point in the interior of $\Pi$ and $1 \leq q \leq \infty$. One defines the $K$ interpolation space $\bar{A}_{(\alpha, \beta), q ; K}$ as the set of $f$ which can be written as $f=f_{0}+\ldots+f_{n}$ with $f_{i} \in A_{i}$ for which

$$
\|f\|_{(\alpha, \beta), q ; K}:=\left(\int_{0}^{\infty} \int_{0}^{\infty}\left(t^{-\alpha} s^{-\beta} K(t, s, f, \bar{A})\right)^{q} \frac{d s}{s} \frac{d t}{t}\right)^{\frac{1}{q}}<\infty .
$$

The function $f \mapsto\|f\|_{(\alpha, \beta), q ; K}$ is used as norm on $\bar{A}_{(\alpha, \beta), q ; K}$. One also defines the $J$-interpolation space $A_{(\alpha, \beta), q ; J}$ as the set of $f$ which can be written as $f=f_{0}+\ldots+f_{n}$ with $f_{i} \in A_{i}$ for which

$$
\|f\|_{(\alpha, \beta), q ; J}:=\inf \left(\int_{0}^{\infty} \int_{0}^{\infty}\left(t^{-\alpha} s^{-\beta} J(t, s, u(t, s), \bar{A})\right)^{q} \frac{d s}{s} \frac{d t}{t}\right)^{\frac{1}{q}}<\infty .
$$


The infimum is taken over all representation of $f$ on the form

$$
f=\int_{0}^{\infty} \int_{0}^{\infty} u(t, s) \frac{d t}{t} \frac{d s}{s} .
$$

In the formula (3) $u$ is strongly measurable with values in $A_{0} \cap \ldots \cap A_{n}$ and the integral is understood as an intersection valued Bochner integral over $[a, b] \times[c, d] \subset(0, \infty) \times(0, \infty)$ and convergence, $a, c \rightarrow 0$ and $b, d \rightarrow \infty$, in $A_{0}+\ldots+A_{n}$ with norm $K(1,1, \cdot, \bar{A})$. The function $f \mapsto\|f\|_{(\alpha, \beta), q ; J}$ is used as a norm on this space. It is a well-known fact that the equivalence theorem does not hold for the polygon method. We only have the embedding $\bar{A}_{(\alpha, \beta), q ; J} \hookrightarrow \bar{A}_{(\alpha, \beta), q ; K}$, see [13]. This implies, in particular, that there is no general form of the reiteration formula. When we write $\bar{A}_{(\alpha, \beta), q}$ we mean either $\bar{A}_{(\alpha, \beta), q ; K}$ or $\bar{A}_{(\alpha, \beta), q ; J}$.

If $R$ is an affine bijection on $R^{2}$, then $\bar{A}_{(\alpha, \beta), q}$ with respect to the polygon $\Pi:=\left[P_{0} \ldots P_{n}\right]$ is equivalent to $\bar{A}_{R(\alpha, \beta), q}$ with respect to $R(\Pi):=\left[R\left(P_{0}\right) \ldots\right.$ $\left.R\left(P_{n}\right)\right]$, see [12].

For triples, with the polygon as the simplex, i.e. $[(0,0)(1,0)(0,1)]$, the polygon method coincides with the Sparr method, see [22], for three spaces and the $K$ functional will be

$$
K(t, s, f, \bar{A}):=\inf \left\{\left\|f_{0}\right\|_{A_{0}}+t\left\|f_{1}\right\|_{A_{1}}+s\left\|f_{2}\right\|_{A_{2}}\right\},
$$

where the infimum is taken over all decomposition $f=f_{0}+f_{1}+f_{2}$, with $f_{i} \in A_{i}$. For this case a Holmstedt type formula for $L_{p}$-spaces and descriptions of some other $K$ functionals have recently been obtained in [14].

For quadruples, with the polygon as the unit square $[(0,0)(1,0)(0,1)$ $(1,1)$, the polygon method coincides the Fernandez method, see [15], for four spaces and the $K$ functional will be

$$
K(t, s, f, \bar{A}):=\inf \left\{\left\|f_{0}\right\|_{A_{0}}+t\left\|f_{1}\right\|_{A_{1}}+s\left\|f_{2}\right\|_{A_{2}}+t s\left\|f_{3}\right\|_{A_{3}}\right\},
$$

where the infimum is taken over all decomposition $f=f_{0}+f_{1}+f_{2}+f_{3}$, with $f_{i} \in A_{i}$.

\section{Two lemmas}

In this section we give two lemmas. These are crucial for the main results of the next section but they are also of independent interest.

First we consider an arbitrary compatible $(n+1)$-tuple. In this lemma it is of course not necessary that we restrict ourselves to just three spaces $B_{i}$. This lemma, in the case when $B_{i}$ is equal to $A_{m_{i}}$, can be found in [10].

Lemma 1. Let $\bar{A}$ be an arbitrary compatible $(n+1)$-tuple of Banach spaces 
and $\Pi$ an arbitrary convex polygon of $\mathrm{R}^{2}$. Suppose $B_{i}$ is placed at, see figure 1 ,

$$
\left(u_{i}, v_{i}\right)=\left(1-\rho_{i}\right)\left(x_{m_{i}}, y_{m_{i}}\right)+\rho_{i}\left(x_{n_{i}}, y_{n_{i}}\right) .
$$

Let $(\alpha, \beta)$ be in the interior of the triangle $\left[\left(u_{1}, v_{1}\right)\left(u_{2}, v_{2}\right)\left(u_{3}, v_{3}\right)\right]$ and $1 \leq q \leq \infty$.

If $B_{i}$ is of class $\mathrm{C}_{K}\left(\rho_{i}, A_{m_{i}}, A_{n_{i}}\right)$ where $0 \leq \rho_{i} \leq 1$, then

$$
\left(B_{1}, B_{2}, B_{3}\right)_{(\alpha, \beta), q ; K} \hookrightarrow \bar{A}_{(\alpha, \beta), q ; K}
$$

and if $B_{i}$ is of class $\mathrm{C}_{J}\left(\rho_{i}, A_{m_{i}}, A_{n_{i}}\right)$ where $0 \leq \rho_{i} \leq 1$, then

$$
\bar{A}_{(\alpha, \beta), q ; J} \hookrightarrow\left(B_{1}, B_{2}, B_{3}\right)_{(\alpha, \beta), q ; J} .
$$

Proof. We have, for all $f \in B_{1}+B_{2}+B_{3}$,

$$
\begin{aligned}
& K(t, s, f, \bar{A})=\inf \left\{\sum_{i=0}^{n} t^{x_{i}} s^{y_{i}}\left\|f_{i}\right\|_{A_{i}}\right\} \\
& \leq \inf \left\{\sum_{i=1}^{3} t^{x_{m_{i}}} S^{y_{m_{i}}} K\left(t^{x_{n_{i}}-x_{m_{i}}} y^{y_{n_{i}}-y_{m_{i}}}, f_{i}, A_{m_{i}}, A_{n_{i}}\right)\right\} \\
& \lesssim \inf \left\{\sum_{i=1}^{3} t^{x_{m_{i}}\left(1-\rho_{i}\right)+x_{n_{i}} \rho_{i}} s^{y_{m_{i}}\left(1-\rho_{i}\right)+y_{n_{i}} \rho_{i}}\left\|f_{i}\right\|_{B_{i}}\right\},
\end{aligned}
$$

and thus

$$
K(t, s, f, \bar{A}) \lesssim K\left(t, s, f, B_{1}, B_{2}, B_{3}\right),
$$

hence (4) holds. For the $J$ functional we have, for all $u \in A_{0} \cap \ldots \cap A_{n}$,

$$
\begin{aligned}
& J(t, s, u, \bar{A})=\max \left\{t^{x_{i}} s^{y_{i}}\|u\|_{A_{i}}\right\} \\
& \geq \max \left\{t^{x_{m_{i}}} S^{y_{m_{i}}} J\left(t^{x_{n_{i}}-x_{m_{i}}} s^{y_{n_{i}}-y_{m_{i}}}, u, A_{m_{i}}, A_{n_{i}}\right)\right\} \\
& \gtrsim \max \left\{t^{x_{m_{i}}\left(1-\rho_{i}\right)+x_{n_{i}} \rho_{i}} s^{y_{m_{i}}\left(1-\rho_{i}\right)+y_{n_{i}} \rho_{i}}\|u\|_{B_{i}}\right\} \text {, }
\end{aligned}
$$

and thus

$$
J(t, s, u, \bar{A}) \gtrsim J\left(t, s, u, B_{1}, B_{2}, B_{3}\right) .
$$

Take an arbitrary element $f \in \bar{A}_{(\alpha, \beta), q ; J}$, and we have to prove that a representation of $f$ of the form (3) with respect to $\bar{A}$ also is a representation with respect to $\left(B_{1}, B_{2}, B_{3}\right)$. Choose an $u$ such that 


$$
f=\int_{0}^{\infty} \int_{0}^{\infty} u(t, s) \frac{d t}{t} \frac{d s}{s}
$$

and

$$
\int_{0}^{\infty} \int_{0}^{\infty}\left[t^{-\alpha} s^{-\beta} J(t, s, u(t, s), \bar{A})\right]^{q} \frac{d t}{t} \frac{d s}{s}<\infty .
$$

Let $C$ be an arbitrary rectangle, or even an arbitrary compact set, in $(0, \infty) \times(0, \infty)$. Since the intersection of $A_{i}$ is continuously embedded in $B_{1} \cap B_{2} \cap B_{3}$ the Bochner integral is also defined when $u$ is viewed as $B_{1} \cap B_{2} \cap B_{3}$-valued. It remains to consider the convergence. We now follow the argument in Remark 4.3 from [22]. By using

$$
\begin{gathered}
K\left(1,1, u(t, s), B_{1}, B_{2}, B_{3}\right) \\
\leq \min \left\{t^{-x_{m_{i}}\left(1-\rho_{i}\right)-x_{n_{i}} \rho_{i}} s^{-y_{m_{i}}\left(1-\rho_{i}\right)-y_{n_{i}} \rho_{i}}\right\} J\left(t, s, u(t, s), B_{1}, B_{2}, B_{3}\right),
\end{gathered}
$$

together with Holder's inequality and (6) we get

$$
\begin{gathered}
\iint_{C} K\left(1,1, u(t, s), B_{1}, B_{2}, B_{3}\right) \frac{d t}{t} \frac{d s}{s} \\
\lesssim\left(\int_{0}^{\infty} \int_{0}^{\infty}\left(t^{\alpha} s^{\beta} \min \left\{t^{-x_{m_{i}}\left(1-\rho_{i}\right)-x_{n_{i}} \rho_{i}} s^{-y_{m_{i}}\left(1-\rho_{i}\right)-y_{n_{i}} \rho_{i}}\right\}\right)^{q^{\prime}} \frac{d t}{t} \frac{d s}{s}\right)^{\frac{1}{q^{\prime}}} \\
\times\left(\int_{0}^{\infty} \int_{0}^{\infty}\left[t^{-\alpha} s^{-\beta} J(t, s, u(t, s), \bar{A})\right]^{q} \frac{d t}{t} \frac{d s}{s}\right)^{\frac{1}{q}} .
\end{gathered}
$$

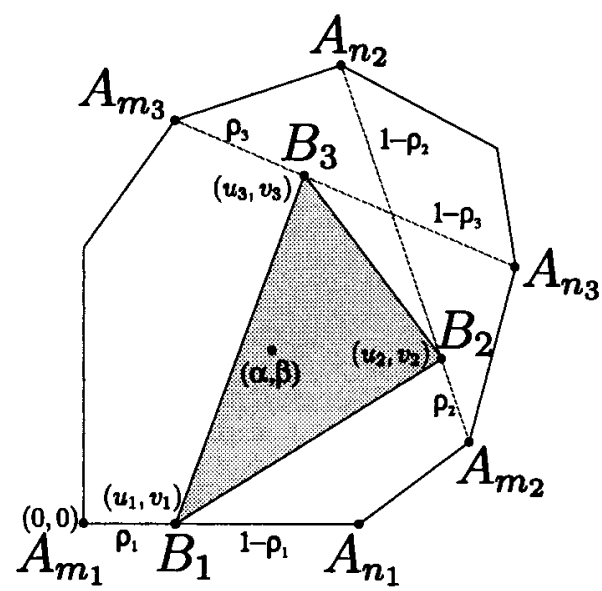

Figure 1: Geometrical illustration of Lemma 1 
The first factor can be seen to be finite. In fact, for $q^{\prime}=1$ this calculation can be found in [13] but the same arguments work for any $q^{\prime} \geq 1$. Hence we have absolute convergence in $B_{1}+B_{2}+B_{3}$ and thus convergence in $B_{1}+B_{2}+B_{3}$ and the proof is complete.

In the second lemma of this section we study $(n+1)$-tuples, $\bar{A}:=\left(A_{0}, A_{1}, \ldots, A_{n}\right)$, where $A_{i}$ is of class $\mathrm{C}\left(\theta_{i}, X, Y\right), 0 \leq \theta_{i} \leq 1$, for a compatible pair $(X, Y)$. Here $n$ is an integer $\geq 2$. We assume that the underlying convex polygon lies in the upper half plane i.e.

$$
\Pi:=\left[\left(x_{0}, y_{0}\right)\left(x_{1}, y_{1}\right) \ldots\left(x_{n}, y_{n}\right)\right],
$$

where $y_{i} \geq 0$. By the invariance under affine bijections this is no restriction.

Lemma 2. Let $(X, Y)$ be a compatible pair of Banach spaces, $(\alpha, \beta)$ an interior point of $\Pi$ and $1 \leq q \leq \infty$. Suppose that

$$
\delta x_{i}+\rho y_{i} \leq \theta_{i} \leq \delta^{\prime} x_{i}+\rho^{\prime} y_{i},
$$

for $\delta \cdot \delta^{\prime}>0$ and $\rho, \rho^{\prime} \neq 0$ and such that $\delta \alpha+\rho \beta$ and $\delta^{\prime} \alpha+\rho^{\prime} \beta$ both are strictly between zero and one. If $A_{i}$ is of class $\mathrm{C}_{K}\left(\theta_{i}, X, Y\right)$ then

$$
\bar{A}_{(\alpha, \beta), q ; K} \hookrightarrow(X, Y)_{\delta \alpha+\rho \beta, q}+(X, Y)_{\delta^{\prime} \alpha+\rho^{\prime} \beta, q},
$$

and if $A_{i}$ is of class $\mathrm{C}_{J}\left(\theta_{i}, X, Y\right)$ then

$$
(X, Y)_{\delta \alpha+\rho \beta, q} \cap(X, Y)_{\delta^{\prime} \alpha+\rho^{\prime} \beta, q} \hookrightarrow \bar{A}_{(\alpha, \beta), q ; J} .
$$

Proof. Let $q<\infty$ and $\rho, \rho^{\prime}>0$. The general case follows by making obvious modifications of the proof below. For $s \geq t$ and $t \leq 1$ we have, for $f \in A_{0}+\ldots+A_{n}$,

$$
\begin{aligned}
K\left(t^{\delta}, s^{\rho}, f, \bar{A}\right) & =\inf \left\{\sum_{i=0}^{n} t^{\delta x_{i}} s^{\rho y_{i}}\left\|f_{i}\right\|_{A_{i}}\right\} \\
& =\inf \left\{\sum_{i=0}^{n} t^{\delta x_{i}+\rho y_{i}}(s / t)^{\rho y_{i}}\left\|f_{i}\right\|_{A_{i}}\right\} \geq \inf \left\{\sum_{i=0}^{n} t^{\theta_{i}}\left\|f_{i}\right\|_{A_{i}}\right\} \\
& \gtrsim \inf \left\{\sum_{i=0}^{n} K\left(t, f_{i}, X, Y\right)\right\}=K(t, f, X, Y),
\end{aligned}
$$

and similarly for $s \geq t$ and $t>1$ we get

$$
K\left(t^{\delta^{\prime}}, s^{\rho^{\prime}}, f, \bar{A}\right) \gtrsim K(t, f, X, Y) .
$$

By these estimates of the $K$ functional and a change of variables we have 


$$
\begin{aligned}
\|f\|_{\bar{A}_{(\alpha, \beta), q ; K}}^{q} & \gtrsim \int_{0}^{1} \int_{t}^{\infty}\left[t^{-\delta \alpha} s^{-\rho \beta} K(t, f, X, Y)\right]^{q} \frac{d s}{s} \frac{d t}{t} \\
& +\int_{1}^{\infty} \int_{t}^{\infty}\left[t^{-\delta^{\prime} \alpha} s^{-\rho^{\prime} \beta} K(t, f, X, Y)\right]^{q} \frac{d s}{s} \frac{d t}{t} .
\end{aligned}
$$

The right hand side is equivalent to

$$
\int_{0}^{1}\left[t^{-\delta \alpha-\rho \beta} K(t, f, X, Y)\right]^{q} \frac{d t}{t}+\int_{1}^{\infty}\left[t^{-\delta^{\prime} \alpha-\rho^{\prime} \beta} K(t, f, X, Y)\right]^{q} \frac{d t}{t},
$$

and which is, by Holmstedt's formula [17] (note that $\delta \alpha+\rho \beta \leq \delta^{\prime} \alpha+\rho^{\prime} \beta$ ), equivalent to the $q$ : th power of $f^{\prime}$ 's norm in $(X, Y)_{\delta \alpha+\rho \beta, q}+(X, Y)_{\delta^{\prime} \alpha+\rho^{\prime} \beta, q}$ and the embedding (8) follows.

We now turn to the $J$-method. Assume $\delta, \delta^{\prime}>0$. As for the $K$ functional, see that for $u \in X \cap Y$ and $s \leq t$

$$
J\left(t^{\delta^{\prime}}, s^{\rho^{\prime}}, u, \bar{A}\right) \lesssim J(t, u, X, Y), \text { for } t \leq 1,
$$

and

$$
J\left(t^{\delta}, s^{\rho}, u, \bar{A}\right) \lesssim J(t, u, X, Y), \text { for } t>1 .
$$

Take an $f \in(X, Y)_{\delta \alpha+\rho \beta, q} \cap(X, Y)_{\delta^{\prime} \alpha+\rho^{\prime} \beta, q}$, we then have by the Fundamental Lemma of Interpolation, see e.g. [3],

$$
J(t, u(t), X, Y) \lesssim K(t, f, X, Y),
$$

for a representation

$$
f=\int_{0}^{\infty} u(t) \frac{d t}{t}
$$

For later use we define

$$
v(t, s):= \begin{cases}\frac{1}{\delta^{\prime}} u\left(t^{\frac{1}{\delta^{\prime}}}\right) & , \text { if } t^{\frac{1}{\delta^{\prime}}} / e \leq s^{\frac{1}{\rho^{\prime}}} \leq t^{\frac{1}{\delta^{\prime}}} \text { and } t \leq 1 \\ \frac{1}{\delta} u\left(t^{\frac{1}{\delta}}\right) & , \text { if } t^{\frac{1}{\delta}} / e \leq s^{\frac{1}{\rho}} \leq t^{\frac{1}{\delta}} \text { and } t>1 \\ 0 & , \text { otherwise, }\end{cases}
$$

this $v$ satisfies

$$
f=\int_{0}^{\infty} \int_{0}^{\infty} v(t, s) \frac{d s}{s} \frac{d t}{t}
$$

We have 


$$
\begin{gathered}
\|f\|_{(X, Y)_{\delta^{\prime} \alpha+\rho^{\prime} \beta, q} \cap(X, Y)_{\delta \alpha+\rho \beta, q}}^{q} \\
\gtrsim \int_{0}^{1}\left[t^{-\delta^{\prime} \alpha-\rho^{\prime} \beta} K(t, f, X, Y)\right]^{q} \frac{d t}{t}+\int_{1}^{\infty}\left[t^{-\delta \alpha-\rho \beta} K(t, f, X, Y)\right]^{q} \frac{d t}{t} .
\end{gathered}
$$

From (11) it follows that

$$
\int_{0}^{1}\left[t^{-\delta^{\prime} \alpha-\rho^{\prime} \beta} K(t, f, X, Y)\right]^{q} \frac{d t}{t} \gtrsim \int_{0}^{1}\left[t^{-\delta^{\prime} \alpha-\rho^{\prime} \beta} J(t, u(t), X, Y)\right]^{q} \frac{d t}{t} .
$$

The integral on the right hand side of this is equivalent to

$$
\int_{0}^{1} \int_{t / e}^{t}\left[t^{-\delta^{\prime} \alpha} s^{-\rho^{\prime} \beta} J(t, u(t), X, Y)\right]^{q} \frac{d s}{s} \frac{d t}{t}
$$

and by using (10), (12) and a change of variables we arrive at

$$
\int_{0}^{1}\left[t^{-\delta^{\prime} \alpha-\rho^{\prime} \beta} K(t, f, X, Y)\right]^{q} \frac{d t}{t} \gtrsim \int_{0}^{1} \int_{0}^{\infty}\left[t^{-\alpha} s^{-\beta} J(t, s, v(t, s), \bar{A})\right]^{q} \frac{d s}{s} \frac{d t}{t} .
$$

In the same way it follows that

$$
\int_{1}^{\infty}\left[t^{-\delta \alpha-\rho \beta} K(t, f, X, Y)\right]^{q} \frac{d t}{t} \gtrsim \int_{1}^{\infty} \int_{0}^{\infty}\left[t^{-\alpha} s^{-\beta} J(t, s, v(t, s), \bar{A})\right]^{q} \frac{d s}{s} \frac{d t}{t} .
$$

Thus we have obtained

$$
\|f\|_{(X, Y)_{\delta, q} \cap(X, Y)_{\nu, q}} \gtrsim \int_{0}^{\infty} \int_{0}^{\infty}\left[t^{-\alpha} s^{-\beta} J(t, s, v(t, s), \bar{A})\right] \frac{d s}{s} \frac{d t}{t},
$$

and the embedding (9) follows for $\delta, \delta^{\prime}>0$. The case when $\delta, \delta^{\prime}<0$ follows in the same way, and the proof is complete.

Remark 1. If $X \cap Y$ is dense in $X, Y$ and in $A_{i}$ and if $1 \leq q<\infty$ then (9) follows from (8) and duality. Indeed, by the duality between the $J$ and $K$ functional it yields that if $A_{i}$ is of class $\mathrm{C}_{J}\left(\theta_{i}, X, Y\right)$ then $A_{i}^{\prime}$ is of class $\mathrm{C}_{K}\left(\theta_{i}, X^{\prime}, Y^{\prime}\right)$. Hence we may apply $(8)$ to the dual $(n+1)$-tuple $\overline{A^{\prime}}$. Now it remains to use classical duality and the polygon duality formula, see [8],

$$
\left(\bar{A}_{(\alpha, \beta), q ; J}\right)^{\prime} \hookrightarrow{\overline{A^{\prime}}}_{(\alpha, \beta), q^{\prime} ; K}
$$

\section{The main results}

In this section we study $(n+1)$-tuples, $\bar{A}:=\left(A_{0}, A_{1}, \ldots, A_{n}\right)$, where $A_{i}$ is of class $\mathrm{C}\left(\theta_{i}, X, Y\right), 0 \leq \theta_{i} \leq 1$, for a compatible pair $(X, Y)$. The underlying polygon is 


$$
\Pi:=\left[\left(x_{0}, y_{0}\right)\left(x_{1}, y_{1}\right) \ldots\left(x_{n}, y_{n}\right)\right],
$$

i.e. the space $A_{i}$ is supposed to be placed on the vertex $\left(x_{i}, y_{i}\right)$. This polygon is fixed. Furthermore, we will consider a polygon

$$
\Pi^{\prime}:=\left[\left(x_{0}^{\prime}, y_{0}^{\prime}\right)\left(x_{1}^{\prime}, y_{1}^{\prime}\right) \ldots\left(x_{n}^{\prime}, y_{n}^{\prime}\right)\right]
$$

where $y_{i}^{\prime} \geq 0$, such that there exists an affine bijection from $\Pi$ onto $\Pi^{\prime}$. The choice of the polygon $\Pi^{\prime}$ depends on $\theta_{i}$. Throughout this section $R$ will the affine bijection that maps $\left(x_{i}, y_{i}\right)$ on $\left(x_{i}^{\prime}, y_{i}^{\prime}\right)$. Here $n$ is an integer $\geq 2$.

The first main theorem reads:

THEOREM 3. Suppose that the assumptions above are satisfied and let $(\alpha, \beta)$ be an interior point of the convex polygon $\Pi$ and let $1 \leq q \leq \infty$.

a) If there exists $\delta \neq 0, \rho \neq 0$ and a polygon $\Pi^{\prime}$ such that

$$
\theta_{i}=\delta x_{i}^{\prime}+\rho y_{i}^{\prime}
$$

for $i=0, \ldots, n$, then

$$
\bar{A}_{(\alpha, \beta), q ; K} \cong \bar{A}_{(\alpha, \beta), q ; J} \cong(X, Y)_{\delta \alpha^{\prime}+\rho \beta^{\prime}, q},
$$

where $R(\alpha, \beta)=\left(\alpha^{\prime}, \beta^{\prime}\right)$.

b) If (13) is not satisfied for any $\delta \neq 0, \rho \neq 0$ and $\Pi^{\prime}$, and $X \cap Y$ is not closed in $X+Y$, then none of the statements $\cong$ in (14) will be true in general.

Remark 2. A special case of Theorem 3 a) was proved in [10]. More exactly, it was proved that for $(\alpha, \beta)$ on the line between two vertices and $A_{i}=(X, Y)_{\theta_{i}, q_{i}}$ we have $\bar{A}_{(\alpha, \beta), 1 ; J} \cong(X, Y)_{\delta \alpha+\rho \beta, 1}$ and $\bar{A}_{(\alpha, \beta), \infty ; K} \cong$ $(X, Y)_{\delta \alpha+\rho \beta, \infty}$.

Proof. a) By the invariance under affine bijection we may assume that $\Pi^{\prime}=\Pi$. Hence the equation (13) holds for the polygon $\Pi$, i.e.

$$
\theta_{i}=\delta x_{i}+\rho y_{i},
$$

and that $y_{i} \geq 0$ and $\left(\alpha^{\prime}, \beta^{\prime}\right)=(\alpha, \beta)$. Since $A_{i}$ is of class $\mathrm{C}\left(\theta_{i}, X, Y\right)$ we can use both embeddings in Lemma 2. The choice of $\delta$ and $\rho$ in (13) satisfies the inequalities in (7), and note that $0<\delta \alpha+\rho \beta<1$. Hence we get

$$
(X, Y)_{\delta \alpha+\rho \beta, q} \hookrightarrow \bar{A}_{(\alpha, \beta), q ; J} \text { and } \bar{A}_{(\alpha, \beta), q ; K} \hookrightarrow(X, Y)_{\delta \alpha+\rho \beta, q}
$$

Now a) is proven since we have the general embedding $\bar{A}_{(\alpha, \beta), q ; J} \hookrightarrow \bar{A}_{(\alpha, \beta), q ; K}$.

b) Consider for example the polygon, $n \geq 3$,

$$
\left[(0,0)(1,0)\left(x_{2}, y_{2}\right) \ldots\left(x_{n-1}, y_{n-1}\right)(0,1)\right] .
$$

Assume that $\theta_{0}=0$ and $\theta_{j} \neq \theta_{1} x_{j}+\theta_{n} y_{j}$ for some $2 \leq j \leq n-1$. In this situation equation (13) cannot hold for any parameters $\delta$ and $\rho$. Let $\alpha+\beta<1$ 
and that $(\alpha, \beta)$ is in the interior of the triangle $\left[(0,0)(1,0)\left(x_{j}, y_{j}\right)\right]$. From Lemma 1 it follows that

$$
\bar{A}_{(\alpha, \beta), q ; J} \hookrightarrow\left(A_{0}, A_{1}, A_{n}\right)_{(\alpha, \beta), q ; J} \cap\left(A_{0}, A_{1}, A_{j}\right)_{(\alpha, \beta), q ; J},
$$

and

$$
\left(A_{0}, A_{1}, A_{n}\right)_{(\alpha, \beta), q ; K}+\left(A_{0}, A_{1}, A_{j}\right)_{(\alpha, \beta), q ; K} \hookrightarrow A_{(\alpha, \beta), q ; K} .
$$

We now use the part a) of this theorem and find that $\left(A_{0}, A_{1}, A_{n}\right)_{(\alpha, \beta), q}$ is equivalent to $(X, Y)_{\theta_{1} \alpha+\theta_{n} \beta, q}$ and $\left(A_{0}, A_{1}, A_{j}\right)_{(\alpha, \beta), q}$ is equivalent to $(X, Y)_{\nu, q}$ where $\nu \neq \delta \alpha+\rho \beta$ since $\theta_{j} \neq \delta x_{j}+\rho y_{j}$. Hence we have obtained

$$
\bar{A}_{(\alpha, \beta), q ; J} \hookrightarrow(X, Y)_{\rho, q} \cap(X, Y)_{\theta_{1} \alpha+\theta_{n} \beta, q},
$$

and

$$
(X, Y)_{\rho, q}+(X, Y)_{\theta_{1} \alpha+\theta_{n} \beta, q} \hookrightarrow \bar{A}_{(\alpha, \beta), q ; K} .
$$

Now, the so called Dependence of Parameter Theorem says that $(X, Y)_{\nu, q} \neq$ $(X, Y)_{\delta \alpha+\rho \beta, q}$ since $X \cap Y$ is not closed in $X+Y$, see [18], and the proof is complete.

REMARK 3. By this theorem we see that embeddings of Lemma 1 can be reversed for some pairs $\bar{A}$. Indeed, let $\Pi$ be a convex polygon in the upper half plane of $\mathrm{R}^{2}$, let $(\alpha, \beta)$ be an interior point of $\Pi$ and $1 \leq q \leq \infty$. Assume $A_{i} \in \mathrm{C}\left(\theta_{i}, X, Y\right)$ for a compatible pair $(X, Y)$ and where $0 \leq \theta_{i} \leq 1$. If there exists $\delta \neq 0$ and $\rho \neq 0$ such that

$$
\theta_{i}=\delta x_{i}+\rho y_{i}, \text { for } i=0, \ldots, n,
$$

then

$$
\bar{A}_{(\alpha, \beta), q} \cong\left(B_{1}, B_{2}, B_{3}\right)_{(\alpha, \beta), q},
$$

where $B_{i}$ is of class $\mathrm{C}\left(\rho_{i}, A_{m_{i}}, A_{n_{i}}\right)$ with $0 \leq \rho_{i} \leq 1$ such that

$$
\left(u_{i}, v_{i}\right)=\left(1-\rho_{i}\right)\left(x_{m_{i}}, y_{m_{i}}\right)+\rho_{i}\left(x_{n_{i}}, y_{n_{i}}\right),
$$

see figure 1. This follows from the previous theorem together with Lemma 1.

We look at Theorem 3 in the special case when the polygon is a triangle. In particular if the triangle is $[(0,0)(1,0)(0,1)]$ we are in the Sparr case for three spaces. The corollary reads:

Corollary 4. Let $A_{i}$ be, for a compatible pair $(X, Y)$, of class $\mathrm{C}\left(\theta_{i}, X, Y\right)$, $0 \leq \theta_{i} \leq 1, i=0,1,2$. If not all the $\theta_{i}$ are equal we have that

$$
\left(A_{0}, A_{1}, A_{2}\right)_{(\alpha, \beta), q} \cong(X, Y)_{\eta, q},
$$


where $\eta=\mu_{0} \theta_{0}+\mu_{1} \theta_{1}+\mu_{2} \theta_{2}$ and $\left(\mu_{0}, \mu_{1}, \mu_{2}\right)$ are the barycentric coordinates of $(\alpha, \beta)$ with respect to the triangle's vertices where the space $A_{i}$ is sitting.

Proof. We let

$$
\Pi^{\prime}:=\left[\left(0, \theta_{0}\right)\left(\theta_{1}-2,2\right)\left(0, \theta_{2}\right)\right],
$$

where $0 \leq \theta_{0} \leq \theta_{1} \leq \theta_{2} \leq 1$ and $\theta_{0}<\theta_{2}$. The polygon $\Pi$ can be mapped onto $\Pi^{\prime}$ by an affine bijection $R$. The equation (13) is satisfied with $\delta=\rho=1$. Now, a simple calculation shows that $\delta \alpha^{\prime}+\rho \beta^{\prime}$ is equal to $\mu_{0} \theta_{0}+\mu_{1} \theta_{1}+\mu_{2} \theta_{2}$ where $\mu_{i}, i=0,1,2$, are the barycentric coordinates of $\left(\alpha^{\prime}, \beta^{\prime}\right)$ with respect to the vertices of the polygon (17). Here $R(\alpha, \beta)=\left(\alpha^{\prime}, \beta^{\prime}\right)$, and it only remains to note that the barycentric coordinates are invariant under affine mappings.

We now turn to the second main theorem. The same type of Banach $(n+1)$-tuple as for the previous theorem are considered, and $\Pi^{\prime}$ is as above. Moreover, by a subtriangle we mean a triangle formed by a triple $\left(B_{1}, B_{2}, B_{3}\right)$ as in Lemma 1 , see figure 1 . The theorem reads:

Theorem 5. Suppose that the assumptions above are satisfied, $(\alpha, \beta)$ in the interior of $\Pi$ and $1 \leq q \leq \infty$. Assume that

$$
\delta x_{i}^{\prime}+\rho y_{i}^{\prime} \leq \theta_{i} \leq \delta^{\prime} x_{i}^{\prime}+\rho^{\prime} y_{i}^{\prime}, i=0, \ldots, n,
$$

for $\delta \cdot \delta^{\prime}>0, \rho, \rho^{\prime} \neq 0$ and a polygon $\Pi^{\prime}$, and that $\left(\alpha^{\prime}, \beta^{\prime}\right)$ lies in the interior of two subtriangles of $\Pi^{\prime}$, one which vertices and corresponding classes gives equality in the first inequality of (18) and the second gives equality in the last inequality of (18).

Then, we have

$$
\bar{A}_{(\alpha, \beta), q ; K} \cong(X, Y)_{\delta \alpha^{\prime}+\rho \beta^{\prime}, q}+(X, Y)_{\delta^{\prime} \alpha^{\prime}+\rho^{\prime} \beta^{\prime}, q},
$$

and

$$
\bar{A}_{(\alpha, \beta), q ; J} \cong(X, Y)_{\delta \alpha^{\prime}+\rho \beta^{\prime}, q} \cap(X, Y)_{\delta^{\prime} \alpha^{\prime}+\rho^{\prime} \beta^{\prime}, q},
$$

where $R(\alpha, \beta)=\left(\alpha^{\prime}, \beta^{\prime}\right)$.

Proof. By the invariance under affine bijection we may assume that $\Pi=\Pi^{\prime}$. Hence the equation (18) holds for the polygon $\Pi$, i.e.

$$
\delta x_{i}+\rho y_{i} \leq \theta_{i} \leq \delta^{\prime} x_{i}+\rho^{\prime} y_{i},
$$

and that $y_{i} \geq 0$ and $\left(\alpha^{\prime}, \beta^{\prime}\right)=(\alpha, \beta)$. The embedding $\hookleftarrow$ of (19) follows by Lemma 1, applied to the two subtriangles, combined with Theorem 3 a). For the reversed embedding we use Lemma 2, note that now $\delta \alpha+\rho \beta$ and $\delta^{\prime} \alpha+\rho^{\prime} \beta$ both are strictly between zero and one. In the same way follows (20) and the proof is complete. 


\section{Interpolation over the unit square}

In this section we interpret the theorems from the previous section in the Fernandez case for four spaces. Hence we assume that the polygon $\Pi$ is the unit square i.e.

$$
\Pi:=[(0,0)(1,0)(0,1)(1,1)] .
$$

The quadruple under consideration is $\left(X, A_{1}, A_{2}, Y\right)$ where $A_{i} \in \mathrm{C}\left(\theta_{i}, X, Y\right)$ for $0<\theta_{i}<1$.

We introduce some notation. Let $(\alpha, \beta)$ be in the unit square and such that $\alpha \neq \beta$ and $\alpha+\beta \neq 1$. Now $(\alpha, \beta)$ lies in the interior of $\Delta \cap \Delta^{\prime}$, where $\Delta$ and $\Delta^{\prime}$ are two different triangles formed by vertices from the unit square. Denote by $\mu_{i}$ and $\mu_{i}^{\prime}, i=0,1,2$, the barycentric coordinates of $(\alpha, \beta)$ with respect to the vertices from $\Delta$ and $\Delta^{\prime}$ respectively. Let $\psi_{i}$ and $\psi_{i}^{\prime}$ be the classes of the spaces sitting on the corresponding vertices.

We have:

TheORem 6. Let $(X, Y)$ be a compatible pair of Banach spaces, let $A_{i}$ be of class $\mathrm{C}\left(\theta_{i}, X, Y\right)$, where $0<\theta_{i}<1, i=1,2$ and let $1 \leq q \leq \infty$.

a) If $\theta_{1}+\theta_{2}=1$, then

$$
\left(X, A_{1}, A_{2}, Y\right)_{(\alpha, \beta), q ; J} \cong\left(X, A_{1}, A_{2}, Y\right)_{(\alpha, \beta), q ; K} \cong(X, Y)_{\delta, q},
$$

where $\delta=\alpha \theta_{1}+\beta \theta_{2}$.

b) With the above notations and if $\theta_{1}+\theta_{2} \neq 1$ then

$$
\left(X, A_{1}, A_{2}, Y\right)_{(\alpha, \beta), q ; K} \cong(X, Y)_{\delta, q}+(X, Y)_{\delta^{\prime}, q},
$$

and

$$
\left(X, A_{1}, A_{2}, Y\right)_{(\alpha, \beta), q ; J} \cong(X, Y)_{\delta, q} \cap(X, Y)_{\delta^{\prime}, q},
$$

where $\delta=\mu_{0} \psi_{0}+\mu_{1} \psi_{1}+\mu_{2} \psi_{2}$ and $\delta^{\prime}=\mu_{0}^{\prime} \psi_{0}^{\prime}+\mu_{1}^{\prime} \psi_{1}^{\prime}+\mu_{2}^{\prime} \psi_{2}^{\prime}$.

Proof. Statement a) is contained in Theorem 3 a), we chose $\delta=\theta_{1}$ and $\rho=\theta_{2}$. We turn to b). Assume $\alpha+\beta<1, \alpha>\beta$ and $\theta_{1}+\theta_{2}<1$. We use Theorem 5, with the subtriangles as

$$
[(0,0)(1,0)(0,1)] \text { and }[(0,0)(1,0)(1,1)] \text {, }
$$

with $\delta=\theta_{1}, \rho=\theta_{2}, \delta^{\prime}=\theta_{1}$ and $\rho^{\prime}=1-\theta_{1}$. Now we only have to rewrite these formulas in the barycentric coordinates notation. The case $\theta_{1}+\theta_{2}>1$ works similarly. The other cases, for $\alpha$ and $\beta$, follows from the general formulas 


$$
\left(B_{0}, B_{1}, B_{2}, B_{3}\right)_{(\alpha, \beta), q}=\left(B_{0}, B_{2}, B_{1}, B_{3}\right)_{(\beta, \alpha), q},
$$

and

$$
\left(B_{0}, B_{1}, B_{2}, B_{3}\right)_{(\alpha, \beta), q}=\left(B_{3}, B_{2}, B_{1}, B_{0}\right)_{(1-\alpha, 1-\beta), q} .
$$

This completes the proof.

Remark 4. If $(\alpha, \beta)$ satisfies $\alpha=\beta$ or $\alpha=1-\beta$ we do not get the equivalence formulas in b) as above. For example for $0<\alpha<\frac{1}{2}$ we only find that

$$
(X, Y)_{\nu, q}+(X, Y)_{\alpha\left(\theta_{1}+\theta_{2}\right), q} \hookrightarrow\left(X, A_{1}, A_{2}, Y\right)_{(\alpha, \alpha), q ; K},
$$

and

$$
\left(X, A_{1}, A_{2}, Y\right)_{(\alpha, \alpha), q ; K} \hookrightarrow(X, Y)_{\alpha\left(\theta_{1}+\theta_{2}\right), q}+(X, Y)_{\alpha, q},
$$

where $\nu$ is an arbitrary number strictly between $\alpha$ and $\alpha\left(\theta_{1}+\theta_{2}\right)$. The proof follows in a similar way as the proof of $b$ ) in Corollary 6 but with the difference that we choose the triangle $[(0,0)(1,0)(\xi, 1)]$ instead of $[(0,0)(1,0)$ $(1,1)]$ for an arbitrary number $0<\xi<1$.

However, (21) if $q=\infty$ and (22) if $q=1$ hold even if $\alpha=\beta$ or $\alpha=1-\beta$. This is seen by, see [10], the general inclusions

$$
\bar{A}_{(\alpha, \beta), 1 ; J} \hookrightarrow\left(A_{i}, A_{j}\right)_{\rho, 1} \hookrightarrow\left(A_{i}, A_{j}\right)_{\rho, \infty} \hookrightarrow \bar{A}_{(\alpha, \beta), \infty ; K},
$$

where $(\alpha, \beta)=(1-\rho)\left(x_{i}, y_{i}\right)+\rho\left(x_{j}, y_{j}\right)$.

We end this section by two explicit examples of Theorem 6. First a quadruple of Lorentz spaces and then a quadruple of weighted Lebesgue spaces. Recall the well-known facts:

i) $\left(L_{p_{0}, q_{0}}, L_{p_{1}, q_{1}}\right)_{\eta, r} \cong L_{s, r}$, where $p_{0} \neq p_{1}, \quad \frac{1}{s}=\frac{1-\eta}{p_{0}}+\frac{\eta}{p_{1}}, \quad 0<\eta<1 \quad$ and $1 \leq r \leq \infty$. Here we define $L_{\infty, r}$ as $L_{\infty}$ for all $r$.

ii) $\left(L_{p}\left(\omega_{0}\right), L_{p}\left(\omega_{1}\right)\right)_{\eta, p} \cong L_{p}(\omega)$, where $0<\eta<1$ and $1 \leq p \leq \infty$ and $\omega=\omega_{0}^{1-\eta} \omega_{1}^{\eta}$. Here $\|f\|_{L_{p}(\omega)}:=\|f \omega\|_{L_{p}}$.

ExAmple 1 . Let $1<p_{i} \leq \infty, \quad 1 \leq q_{i} \leq \infty, \quad p_{0}<p_{i}<p_{3}, \quad i=1,2$ and $1 \leq q \leq \infty$.

a) If $\frac{1}{p_{0}}+\frac{1}{p_{3}}=\frac{1}{p_{1}}+\frac{1}{p_{2}}$, then

$$
\left(L_{p_{0}, q_{0}}, L_{p_{1}, q_{1}}, L_{p_{2}, q_{2}}, L_{p_{3}, q_{3}}\right)_{(\alpha, \beta), q} \cong L_{p, q},
$$

where

$$
\frac{1}{p}=\frac{1-\alpha-\beta}{p_{0}}+\frac{\alpha}{p_{1}}+\frac{\beta}{p_{2}} .
$$


b) If $\frac{1}{p_{0}}+\frac{1}{p_{3}} \neq \frac{1}{p_{1}}+\frac{1}{p_{2}}, \alpha+\beta<1$ and $\alpha>\beta$, then

$$
\left(L_{p_{0}, q_{0}}, L_{p_{1}, q_{1}}, L_{p_{2}, q_{2}}, L_{p_{3}, q_{3}}\right)_{(\alpha, \beta), q ; K} \cong L_{\bar{p}, q}+L_{\tilde{p}, q},
$$

and

$$
\left(L_{p_{0}, q_{0}}, L_{p_{1}, q_{1}}, L_{p_{2}, q_{2}}, L_{p_{3}, q_{3}}\right)_{(\alpha, \beta), q ; J} \cong L_{\bar{p}, q} \cap L_{\tilde{p}, q},
$$

where

$$
\frac{1}{\bar{p}}=\frac{1-\alpha-\beta}{p_{0}}+\frac{\alpha}{p_{1}}+\frac{\beta}{p_{2}},
$$

and

$$
\frac{1}{\widetilde{p}}=\frac{1-\alpha}{p_{0}}+\frac{\alpha-\beta}{p_{1}}+\frac{\beta}{p_{3}} .
$$

In [11] weighted Lebesgue spaces are considered. Their results there are for the interpolation spaces

$$
\left(L_{1}\left(\omega_{0}\right), \ldots, L_{1}\left(\omega_{n}\right)\right)_{(\alpha, \beta), 1},
$$

and

$$
\left(L_{\infty}\left(\omega_{0}\right), \ldots, L_{\infty}\left(\omega_{n}\right)\right)_{(\alpha, \beta), \infty} .
$$

Here we have arbitrary $L_{p}$ but not arbitrary weights.

ExAmple 2. Let $1 \leq p \leq \infty$ and let $\omega_{0}$ and $\omega_{1}$ be two arbitrary weights. Define $\bar{\omega}:=\omega_{0}^{1-\theta_{1}} \omega_{1}^{\theta_{1}}$ and $\widetilde{\omega}:=\omega_{0}^{1-\theta_{2}} \omega_{1}^{\theta_{2}}$.

a) If $\theta_{1}+\theta_{2}=1$, then

$$
\left(L_{p}\left(\omega_{0}\right), L_{p}(\bar{\omega}), L_{p}(\widetilde{\omega}), L_{p}\left(\omega_{1}\right)\right)_{(\alpha, \beta), p} \cong L_{p}(\omega),
$$

where

$$
\omega:=\omega_{0}^{1-\alpha \theta_{1}-\beta \theta_{2}} \omega_{1}^{\alpha \theta_{1}+\beta \theta_{2}} .
$$

b) If $\theta_{1}+\theta_{2} \neq 1, \alpha+\beta<1$ and $\alpha>\beta$, then

$$
\left(L_{p}\left(\omega_{0}\right), L_{p}(\bar{\omega}), L_{p}(\widetilde{\omega}), L_{p}\left(\omega_{1}\right)\right)_{(\alpha, \beta), p ; K} \cong L_{p}(\omega),
$$

and

$$
\left(L_{p}\left(\omega_{0}\right), L_{p}(\bar{\omega}), L_{p}(\widetilde{\omega}), L_{p}\left(\omega_{1}\right)\right)_{(\alpha, \beta), p ; J} \cong L_{p}(\widehat{\omega}),
$$

where 


$$
\omega:=\omega_{0}^{1-\alpha \theta_{1}} \omega_{1}^{\alpha \theta_{1}} \min \left\{\left(\frac{\omega_{1}}{\omega_{0}}\right)^{\beta \theta_{2}},\left(\frac{\omega_{1}}{\omega_{0}}\right)^{\beta\left(1-\theta_{1}\right)}\right\},
$$

and

$$
\widehat{\omega}:=\omega_{0}^{1-\alpha \theta_{1}} \omega_{1}^{\alpha \theta_{1}} \max \left\{\left(\frac{\omega_{1}}{\omega_{0}}\right)^{\beta \theta_{2}},\left(\frac{\omega_{1}}{\omega_{0}}\right)^{\beta\left(1-\theta_{1}\right)}\right\} .
$$

\section{A concluding reiteration result}

In this section we will look at reiteration from a more classical point of view. We consider two convex polygons

$$
\Pi:=\left[\left(x_{0}, y_{0}\right)\left(x_{1}, y_{1}\right) \ldots\left(x_{n}, y_{n}\right)\right],
$$

and

$$
\Pi^{\prime}:=\left[\left(\alpha_{0}, \beta_{0}\right)\left(\alpha_{1}, \beta_{1}\right) \ldots\left(\alpha_{m}, \beta_{m}\right)\right],
$$

where $\Pi^{\prime}$ lies in the interior of $\Pi$. The idea is to compare $\bar{A}_{(\alpha, \beta), q}$ with $\bar{B}_{(\alpha, \beta), q}$ where $B_{i}$ is e.g. $\bar{A}_{\left(\alpha_{i}, \beta_{i}\right), q_{i}}$. A result of this type was obtained in [7]. The theorem there deals with the case when $\Pi$ can be mapped onto $\Pi^{\prime}$ by an affine bijection, in particular $m=n$. Our result Proposition 6 a) corresponds to the result in [7] and the part b) combines part a) with Theorem 3 .

We need the concept of classes in the polygon case. Let $\bar{A}$ be the $(n+1)$ tuple $\left(A_{0}, \ldots, A_{n}\right)$. Let $B$ be an intermediate space i.e.

$$
A_{0} \cap \ldots \cap A_{n} \hookrightarrow B \hookrightarrow A_{0}+\ldots+A_{n},
$$

where the intersection is normed by $J(1,1, \cdot, \bar{A})$ and the sum by $K(1,1, \cdot, \bar{A})$. For an arbitrary point $(\alpha, \beta)$ is an interior point of $\Pi$ the space $B$ is said to be of class $\mathrm{C}_{K}(\alpha, \beta, \bar{A})$ if

$$
K(t, s, f, \bar{A}) \lesssim t^{\alpha} s^{\beta}\|f\|_{B}, \text { for all } f \in B,
$$

and of class $\mathbf{C}_{J}(\alpha, \beta, \bar{A})$,

$$
t^{\alpha} s^{\beta}\|u\|_{B} \lesssim J(t, s, u, \bar{A}), \text { for all } u \in A_{0} \cap \ldots \cap A_{n} .
$$

If a space is both of class $\mathrm{C}_{K}(\alpha, \beta, \bar{A})$ and $\mathrm{C}_{J}(\alpha, \beta, \bar{A})$ we say that it is of class $\mathrm{C}(\alpha, \beta, \bar{A})$, in particular $\bar{A}_{(\alpha, \beta), q}$ is of class $\mathrm{C}(\alpha, \beta, \bar{A})$.

Proposition 7. Let $B_{i}$ be of class $\mathrm{C}\left(\alpha_{i}, \beta_{i}, \bar{A}\right), i=0, \ldots, m,(\alpha, \beta)$ an interior point of $\Pi^{\prime}$ and $1 \leq q \leq \infty$.

a) If $B_{i}$ is of class $\mathrm{C}_{K}\left(\alpha_{i}, \beta_{i}, \bar{A}\right)$ then 


$$
\bar{B}_{(\alpha, \beta), q ; K} \hookrightarrow \bar{A}_{(\alpha, \beta), q ; K},
$$

and if $B_{i}$ is of class $\mathrm{C}_{J}\left(\alpha_{i}, \beta_{i}, \bar{A}\right)$ then

$$
\bar{A}_{(\alpha, \beta), q, J} \hookrightarrow \bar{B}_{(\alpha, \beta), q, J} .
$$

b) Assume that $\Pi$ lies in the upper half plane i.e. $y_{i} \geq 0$. If $A_{i}$ is of class $\mathrm{C}\left(\theta_{i}, X, Y\right)$ for a compatible pair $(X, Y)$ and where $0 \leq \theta_{i} \leq 1$ and if there exists $\delta \neq 0$ and $\rho \neq 0$ such that

$$
\theta_{i}=\delta x_{i}+\rho y_{i}, \text { for } i=0, \ldots, n,
$$

then we have

$$
\bar{A}_{(\alpha, \beta), q} \cong \bar{B}_{(\alpha, \beta), q} .
$$

Proof. a) We note that if $B_{i}$ is of class $\mathrm{C}_{K}\left(\alpha_{i}, \beta_{i}, \bar{A}\right)$ then

$$
K(t, s, f, \bar{A}) \lesssim K(t, s, f, \bar{B}), \text { for all } f \in B_{0}+\ldots+B_{n},
$$

and if $B_{i}$ is of class $\mathrm{C}_{J}\left(\alpha_{i}, \beta_{i}, \bar{A}\right)$ then

$$
J(t, s, u, \bar{B}) \lesssim J(t, s, u, \bar{A}) \text {, for all } u \in A_{0} \cap \ldots \cap A_{n} .
$$

If we use this as in the proof of Lemma 1 the embeddings (23) and (24) follows. We turn to b). By Theorem 3 we have that $\bar{A}_{(\alpha, \beta), q, K} \cong \bar{A}_{(\alpha, \beta), q, J}$. Now (25) follows from (23), (24) and the general embedding $\bar{B}_{(\alpha, \beta), q, J} \hookrightarrow \bar{B}_{(\alpha, \beta), q, K}$.

Acknowledgement. I would like to thank the Professors Lech Maligranda and Lars-Erik Persson for their generous advice and encouragement during the work of this paper. I also want to thank Professor Maria J. Carro who has given many valuable comments which have improved the final version of this paper.

\section{REFERENCES}

1. I.U. Asekritova and N.Ya. Krugljak, On equivalence of the $K$ - and $J$-methods for $(N+1)$ tuples of Banach spaces, Studia Math. 122 (1997), 99-116.

2. C. Bennett and R. Sharpley, Interpolation of Operators, Pure Appl. Math. 129. Academic Press, Boston, 1988

3. J. Bergh and J. Löfström, Interpolation Spaces, Springer Verlag, Berlin-Heidelberg-New York, 1976.

4. Yu.A. Brudnyi and N.Ya. Krugljak, Interpolation Functors and Interpolation Spaces, NorthHolland, Amsterdam, 1991.

5. M. Carro, L.I. Nikolova, J. Peetre, and L.E. Persson, Some real interpolation methods for families of Banach spaces - a comparison, J. Approx. Theory 89 (1997), $26-57$.

6. F. Cobos, On the optimality of compactness results for interpolation methods associated to polygons, Indag. Math. 5 (1994), 397-401. 
7. F. Cobos and P. Fernández-Martínez, Reiteration and a Wolff theorem for interpolation methods by means of polygons, Studia Math. 102 (1992), 239-256.

8. F. Cobos and P. Fernández-Martínez, A duality theorem for interpolation methods associated to polygons, Proc. Amer. Math. Soc. 121 (1994), 1093-1101.

9. F. Cobos and P. Fernández-Martínez, Dependence on parameters in interpolation methods associated to polygons, Boll. Un. Mat. Ital. (to appear).

10. F. Cobos, P. Fernández-Martínez, and A. Martínez, On the behaviour of weak compactness under certain interpolation methods, Preprint (1996).

11. F. Cobos, P. Fernández-Martínez, and T. Schonbek, Norm estimates for interpolation methods defined by means of polygons, J. Approx. Theory 80 (1995), 321-351.

12. F. Cobos, T. Kühn, and T. Schonbek, One-sided compactness results for Aronszajn-Gagliardo functors, J. Funct. Anal. 106 (1992), 274-313.

13. F. Cobos and J. Peetre, Interpolation of compact operators: the multidimensional case, Proc. London Math. Soc. 63 (1991), 371-400.

14. S. Ericsson, Description of some $K$ functionals for three spaces and reiteration, Math. Nachr., to appear.

15. D.L. Fernández, Interpolation of $2^{n}$ Banach spaces, Studia Math. 45 (1979), 175-201.

16. P. Fernández-Martínez, Métodos de interpolación asociados a polígonos, $\mathrm{PhD}$ thesis, Universidad de Murcia, Spain, 1994.

17. T. Holmstedt, Interpolation of quasi-normed spaces, Math. Scand. 26 (1970), 177-190.

18. S. Janson, P. Nilsson, J. Peetre, and M. Zafran, Notes on Wolff's note on interpolation spaces, Proc. London Math. Soc. 48 (1984), 283-299.

19. S.G. Krein, Yu.I. Petunin, and E.M. Semenov, Interpolation of Linear Operators, Transl. Math. Monogr. Amer. Math. Soc. 54, Providence, 1982.

20. L. Maligranda, The K-functional for symmetric spaces, Lecture Notes in Math. 1070 (1984), 169-182.

21. L. Maligranda, A bibliography on "Interpolation of Operators and Applications" 1926-1990, Dept. of Math., Luleå University, 1990.

22. G. Sparr, Interpolation of several Banach spaces, Ann. Mat. Pura. Appl. 99 (1974), 247-316.

MATEMATISKA INSTITUTIONEN

LULEÅ UNIVERSITEIT

97187 LULEÅ

SWEDEN

Email: sen@sm.luth.se 